\title{
IGA NEPHROPATHY AS THE INITIAL PRESENTATION IN A PATIENT WITH SYSTEMIC SCLEROSIS: CASE REPORT
}

\author{
Isadora Cristina da Silva ${ }^{1}$, Árien Eliza Oldoni ${ }^{1}$, Diego Ustárroz Cantali²,* \\ 1.Universidade Regional Integrada do Alto Uruguai e das Missões, Erechim (RS), Brazil.; 2.Pontifícia Universidade Católica do Rio Grande \\ do Sul, Porto Alegre (RS), Brazil. \\ *Corresponding author: diegocantali@gmail.com
}

\section{BACKGROUND}

Systemic sclerosis (SSC) is a systemic autoimmune rheumatic disease characterized by fibrosis of the skin and the internal organs associated with vasculopathy. Renal involvement occurs in 60-80\% of patients with SSc, especially in those with the diffuse form of the disease. IgA nephropathy as the initial presentation of systemic sclerosis is rare. Thus, a case of SSc associated with IgA nephropathy was reported.

\section{CASE REPORT}

A 42-year-old female patient, previously diagnosed only with systemic arterial hypertension, showed an abrupt decline in the renal function. Renal biopsy identified IgA nephropathy. On evaluation with a rheumatologist, the patient reported restricted mouth opening, dysphagia, xerostomia, and dyspnea on medium exertion. On physical examination, she presented thinning of the upper lip and nose, Raynaud's phenomenon, puffy hands, sclerodactyly, telangiectasias in the hands and face, and small perioral wrinkles. Complementary investigation showed a mixed antinuclear antibody pattern of nuclear fine speckled and nucleolar pattern (BAC-1) with a titer of 1:640, high titer anti-topoisomerase I antibodies (240), creatinine 1.71 (GFR: 36), spot urine protein-to-creatine ratio (UPCR) 0.2, and qualitative urine analysis showed proteinuria and hematuria. Spirometry indicated a moderate restrictive pattern, and high-resolution computed tomography of the chest showed areas of ground glass opacities in a peripheral distribution predominantly in the lower lobes (interstitial lung disease), affecting more than $20 \%$ of both lungs, and esophageal distension. The patient underwent three monthly cycles of cyclophosphamide of $1 \mathrm{~g}$ each. Control tests showed stabilization of interstitial lung disease and improvement in renal function with a creatinine of 1.32 (GFR: 50) and UPCR 0.01.

\section{CONCLUSION}

Systemic sclerosis is a heterogeneous disease whose clinical presentation is variable due to the involvement of different internal organs. IgA nephropathy as the first manifestation of SSc is rare, and the treatment with an immunosuppressant is not well established. Our case showed a great response with immunosuppressive treatment. Thus, further studies with this association should be carried out to establish the benefits of immunosuppression in this pathology.

\section{KEYWORDS}

IgA nephropathy, Systemic sclerosis, Immunosuppressant. 